INTERNATIONAL DESIGN CONFERENCE - DESIGN 2018

https://doi.org/10.21278/idc.2018.0153

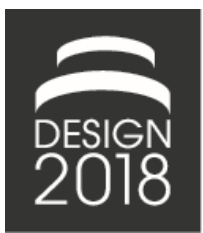

\title{
IDEAL IDEATION: A FRAMEWORK FOR THE MANAGEMENT OF SKETCH INHIBITION AMONG UNDERGRADUATE DESIGNERS
}

\author{
L. Thurlow and P. Ford
}

\begin{abstract}
Sketch inhibition among undergraduates is a growing but under-researched issue. Affected students avoid engaging with sketching during concept development, they also have little understanding of the benefits it offers effective ideation as a language and a cognitive tool which in turn affects design quality and impedes commercial activity. Causes include skill-set shortage, prevalence of technology and social and educational issues. The proposed management framework aims to reduce inhibition via a three-part tool for use by higher education at strategic, pedagogic and individual levels.
\end{abstract}

Keywords: sketch inhibition, design education, conceptual design, design methodology, design research

\section{Introduction}

In a recent interview, Jan Faulkner, (2017) director of Haley Sharpe Design commented that, of the many new graduates he sees during the recruitment process, "less than five per-cent have good drawing skills." Similarly, Martin Witts, (2017) of Sosna Witts Design stated, "I've never met a good designer who couldn't draw." Ironically, back in 2005, Schenk (2005) suggested that the inability of secondary education to equip pupils with the drawing skills essential for higher education was resulting in graduates embarking on their careers with increasingly poor drawing ability and consequently having a negative impact upon design organisations.

Although sketch inhibition is regularly mooted within higher education (HE), its effects on industry have yet to be resolved. With this mind, this paper considers the phenomenon of sketch inhibition and proposes a conceptual pedagogical framework for its management within HE. Because of the relatively unexplored nature of sketch inhibition, this study is indicative as opposed to exhaustive: the research, analysis and theory development is still ongoing, and this paper forms a discussion document of the research to date.

Due to the lack of formal research into sketch inhibition, the underpinning for this study is based upon the limited theory of sketch inhibition already developed by Booth et al. (2016) who identify individual, social and technological causal factors: this together with design process theory, and that of design sketching. Historically, theoretical models of design have presented an often linear process and refer heavily to the analysis and synthesis of information. Although, for example, Koburg and Bagnall (1972), (from Bagnall and Koberg, 1990), offered a revised concept of their initial two step theory, Mesarovic (1964), (from Dubberly, 2004), demonstrates feedback loops and Alexander (1964) demonstrates levels of self-consciousness in his model, these do not consider, to the extent to which they are relied upon, the cognitive and feedback activities for which sketching is the most effective tool. 
Development in the understanding of the convergent and divergent aspects of the design process is more recently modelled by Cross (2000) and Benathy (2000) but these also fail to consider designer cognition. Theories of the micro-functions within design, e.g.; Goldschmidt's linkography (2014) and Suwa and Tversky's (1997) conceptual dependencies model effectively demonstrate the smallest of design moves, do not specifically consider the role of sketching as support for that process. Ferguson's (1992) and Pei et al.'s (2011) taxonomies offer the most developed examples of the types and positions of sketching activity in the design process, but fail to address the combination of behaviour and cognition activities associated with this.

Cash and Kreye's (2017) theory of Uncertainty Driven Action, (UDA) shows design process being driven by uncertainty perception embodied by information action, knowledge sharing action and representation action, (i.e.; sketching), and is valuable as an underpinning to this study. The position of sketching, (and by default, sketch inhibition), is not defined explicitly, but based upon the literature already reviewed it is clear this would sit comfortably within (and prior to, in the case of sketch inhibition) the domain of representation action - this is demonstrated in Figure 1. The lack of explicit theory relating to sketch inhibition, together with an abundance of empirical information lamenting it from both industry and educators is from where the research need has been identified.

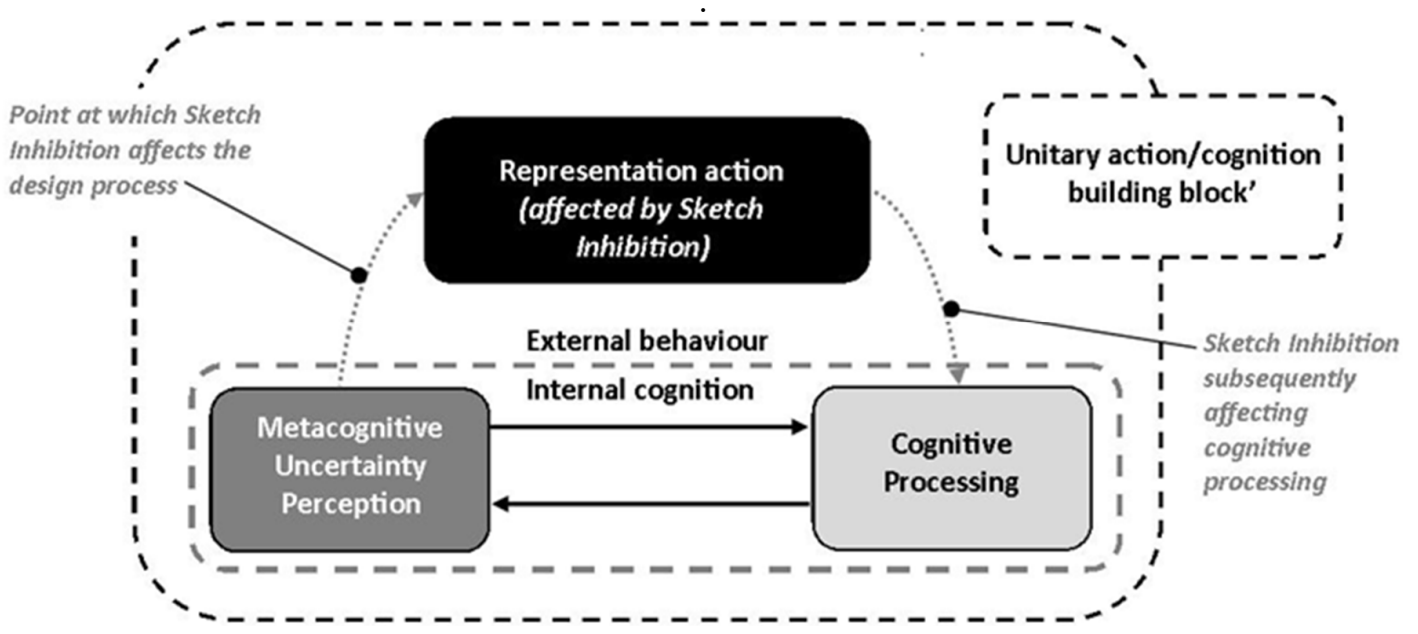

Figure 1. Cash and Kreye's (2017) model of Uncertainty Driven Action (UDA) used to illustrate the potential position and effects of sketch inhibition

\section{Methodology}

The research question has been informed by observations gathered over an 18 year period of teaching within HE - these are also used throughoout this paper to support the findings. Because of the limited theory relating to design sketching and sketch inhibition, the study has favoured a Grounded Theory approach, with particular acknowledgement to the Straussian (Strauss and Corbin, 1990) model. This considers literature to be an acceptable component in development of emergent theory, (which was a vital part of undertaking this study), and necessary, according to Wacker's (1998) definition of theorybuilding, unlike Glaser's (1992) approach which favours no literature.

Development of an emergent theory of sketch inhibition has been sought, and the use of concurrent analysis and theoretical sampling has been a key feature of the approach. The literature has been reviewed, initially focussing on sketch inhibition, however, the dearth of such material led to a need to access a wider sample to contextualise and inform the research.

The literature has been coded using an emergent strategy, referring using Nvivo and has identified the definitions, purpose, micro-processes, and cognitive issues of sketching within the design process. This and the limited literature relating to sketch inhibition provided a basis from which to develop questions - to further "interrogate" (Strauss and Corbin, 1990) the data using a semi-structured interview method. A purposive sampling strategy was used to identify participants for the piloting stage: although not a 
pure Grounded Theory approach, it prevented the wasting of much time and allowed for honing-in on the most useful subjects. The sample comprised educators, (within HE), industry influencers, and third year under-graduate design students from a range of design disciplines identified by their tutors as being sketch inhibited.

The literature along with the observational evidence was used to inform the structure of the initial pilot interviews. During the piloting process, several issues were identified and acted upon, as per the concurrent analysis/theoretical sampling approach favoured by Strauss (Strauss and Corbin, 1990). It was evident that some of the student sample had learning differences: whether these were causal of their sketch inhibition was of interest and it was deemed that a further survey of learning style would be of benefit. Some of the student responses to interview evidenced potential issues of their future employability due to their inhibition - this was considered another emerging factor that would benefit further investigation using a longitudinal study approach; both of these tools are currently in the datagathering stage. As a result of the piloting, it was considered that for comparison, un-inhibited students would also be recruited for interview. This would provide the study with a fuller understanding of the sketch inhibition, including whether it is a binary or scalar phenomenon. The research methodology is modelled in Figure 2.

Educators (including academics), from various disciplines were selected based on their understanding of sketch inhibition. These included authors of the sketch inhibition literature as further interrogation of the issues they presented was considered necessary. Industry influencers, i.e.; those responsible for the recruitment of newly graduated designers were recruited from various disciplines. This data is currently being gathered but in due course, will be presented according to established protocols - however for the purpose of this paper, it is being used informally to reinforce the literature findings. At present, the literature has been reviewed and interviews are ongoing: this study is neither complete nor conclusive and this must be borne in mind: the development of the conceptual pedagogic framework requires the research to be complete (according to the concept of saturation), and at this stage is also presented tentatively.

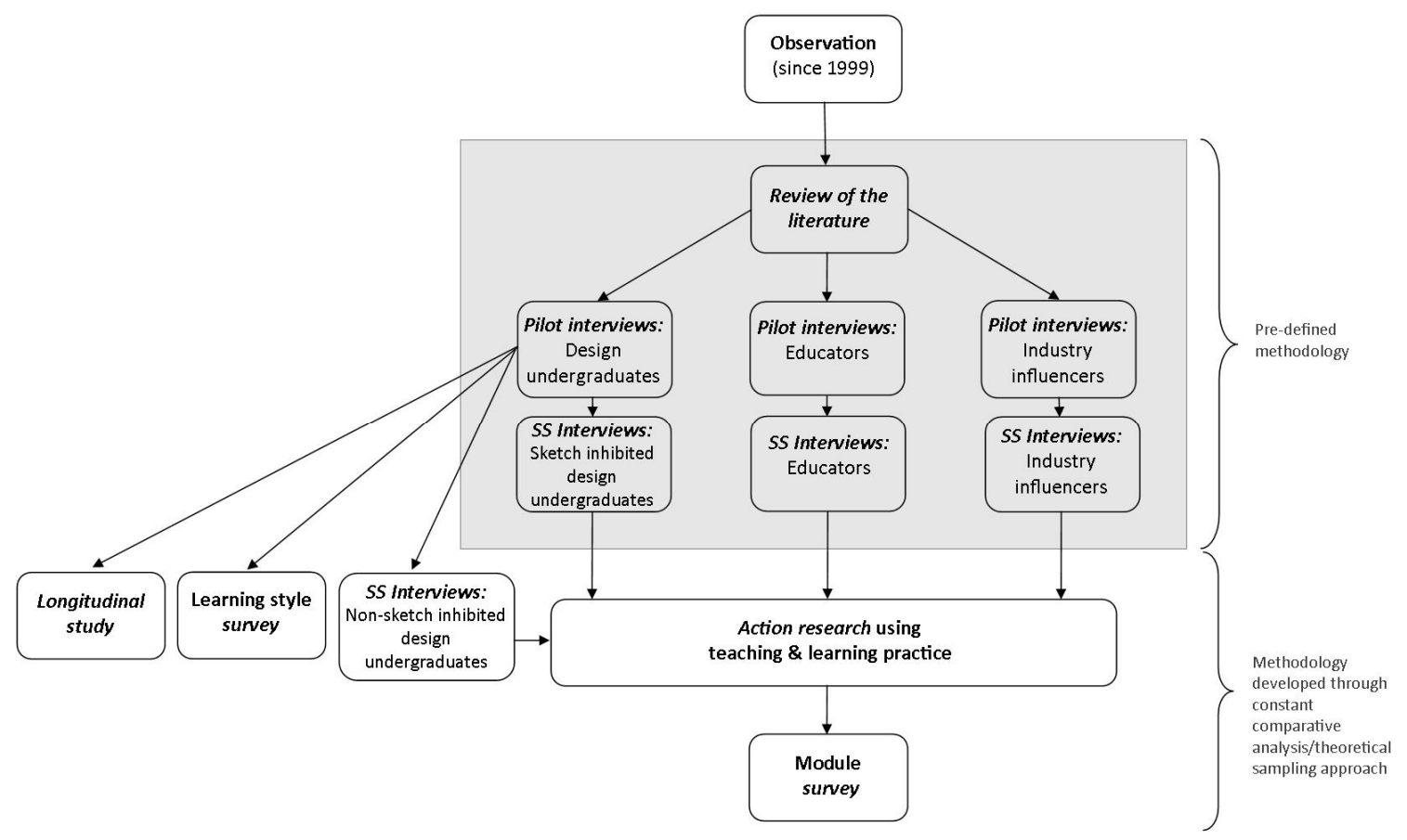

Figure 2. Model of the research methodology

The following sections are presented to contextualise sketching within design activity and establish sketch inhibition within that activity. The initial coding (open coding) of literature enabled themes to be identified, and this forms the structure of the following: The language of sketching is considered in 
Section 3, and its subsequent benefits to the design process in Section 4. The cognitive functions and micro-processes within design are presented in Section 5 and Section 6 respectively. From this, the existence of sketch inhibition is confirmed, (Section 7) and the proposed pedagogic tool is developed (Section 8) and discussed in Section 9.

\section{Sketching as a language within the design process}

Understanding sketch inhibition and its management being the aim of this study, it is has been vital to prove that sketching is intrinsic to the design process. Bilda et al. (2006) established that it enables a dialogue, the opportunity to see, opportunities for re-representation and externalisation of mental imagery. Ferguson's (1992) taxonomy describes sketches as thinking, talking and prescriptive tools. Pei et al. (2011) position sketches, additionally, as tools for persuasion, memory and manufacture.

The ability of sketches to communicate between individuals and across groups suggests that they possess a similar structure to that of language. This progression forms a dialogue between designer and substrate, and creates a language of (and shared by) designers. Stones and Cassidy (2010) suggest that, "a complex, bi-directional cognitive process occurs as the designer sketches" (p. 440). They refer to Schön, (1983) describing ideation sketching as a "conversation" (p. 76) between the designer and the marks he makes. They further compare this to Laseau (2001) who states that, "the process of graphic thinking can be seen as a conversation with ourselves in which we communicate with sketches" (p. 7). Fish and Scrivener (1990) consider this function in the relationship between descriptive and depictive information created during the design process. This language of sketching enables the designer to create mental images based upon what they see on the page as their ideas are recorded. At the micro-level of language, Barthes' semiotic theory can be equally applied to sketches: he considers the literal image to be a denotation of the abstract form - the symbolic image demonstrating that implied through either idiolect or wider language. He suggests that "all images are polysemous; they imply, underlying their signifiers, a floating chain of signifieds, the reader able to choose some and ignore others" (Barthes, 1977, pp. 3839). This can be applied comfortably to the sketch; its ability to furnish the designer with pieces of interconnected and free-standing information within a single image. Eckert et al. (2010) note similarities of experience between design disciplines during the Across Design Project: "we observed appreciative surprise among our informants as they recognized the degree to which the experience of other professionals, who they might not have considered as natural peers, did in fact extend across design" ( $\mathrm{p}$. 30). They identify sketching to develop structure and process and to convey these to others during their respective design process: "Designers are engaged in many of the same activities and concerns, but in very different guises according to their particular technical domains and social or business contexts," (p. 38).

\section{The characteristics of sketches}

The benefits sketching during design development are continually impressed upon students, but rarely considered in greater depth. Speed of the medium is clearly a vital function for the designer: Pei et al. (2010) observe that personal sketches enable the designer "to externalise his thoughts quickly" (p. 12). Powell, M. (2017) believes it offers evidence of thinking, speed of transmission and objective realism that no other medium can. Faulkner (2017) suggests additionally, the need for financial viability in creative business, requiring a designer to be able to offer, "speed of thought, and sketching is part of that." The economy of sketching is considered by Goldschmidt (2003) who suggests that no cognitive energy is used in converting marks on the page into comprehendible information. She also refers to the ability of the sketch to provide an accessible and logical reference to complete sets of developmental information, the entire design process being, by default, a set of serial information.

Reinterpretation of sketch images is an important part of the development of concepts. Schön states that designers are involved in a "transactional," relationship with the design and are in a "reflective conversation with the situation" (Schön, 1992, p. 4) for which "the act of drawing can be rapid and spontaneous, but the residual traces are stable...the graphic world of the sketchpad is the medium of reflection-in-action" (Schön, 1983, p. 153). Goel (1995) also identifies reinterpretation as a vital function of sketches, allowing the designer to apply new meaning to an existing set of imagery. Fish and Scrivener (1990) suggest their ambiguity can enable perception of more than one possibility at a 
time, initiating further mental imagery. They contrast this with that of the computer where the designer can be coerced into early finalising of designs, detailing ill-resolved concepts into poorly resolved solutions: "by failing to represent the important mental component of early sketches, many computer systems have a detrimental effect on the growth of mental imagery in inexperienced students" (p. 118). Crismond and Adams (2012) warn that fixation - the inability of the designer to see beyond a concept towards a more appropriate solution - is also an issue. Atilola et al. (2016) suggest that such fixation negatively affects the design process and its causes are, according to Crismond and Adams (2012), divergent and overlapping. Goldschmidt (2003) makes a strong case for sketching as a tool for its management: "the expert sketcher therefore is someone who can make and manipulate representations fast and with great ease while choosing the most appropriate projection(s). If he or she is a designer, this skill is indispensable in the search that is part of, indeed the most significant part of, the design process" (p. 82). Schön and Wiggins (1992) also consider experience as vital to effective problem-solving, observing that a lack of experience can create working memory limitations for the designer that can be addressed through the use of sketches.

\section{Cognitive support}

The effectiveness of the sketching tool relies on both behavioural and cognitive input, as illustrated by Cash and Kreye's (2017) model. According to Fish and Scrivener (1990), this includes external references gathered from memory and via observation, the result of this being "a conscious stream of thought transferred onto marks on paper" (Witts, 2017) allowing for "access to your interior dialogues in a tangible form to see if they are actually fit for purpose" (Downs, 2017). The importance of the dialogue between designer and sketch is endorsed by Cross: "the thinking processes of the designer seem to hinge around the relationship between internal mental processes and their external expression and representation in sketches" (1999, p. 30).

The tacit functions of the sketch include freeing-up of working memory during complex mental tasks. "Empirical studies on visuo-spatial working memory show that the capacity of the visuo-spatial working memory is limited when visuo-spatial tasks are done using imagery" (Bilda and Gero, 2005, p. 145). This suggests that without the sketch as a cognitive support tool, the designer will struggle with the quantity of complex information that must be handled during ideation: "externalization is needed to offload the visuo-spatial working memory. For the same reason drawings and diagrams play an important role in designing" (Bilda and Gero, 2005, p. 145), thus endorsing sketching as the most appropriate means to offload this.

\section{Micro processes}

Progression through complex explicit and tacit information during design development has been shown to benefit from the cognitive support offered by sketching. Cross (2001) refers to Akin and Akin (1996) regarding the design problem-solving process and suggesting that, for new concepts to be created, the designer's frame of reference needs to be broken, a shift in mode between drawing, examining and thinking allowing the designer to identify new ideas.

Goldschmidt (1991) presents experiments conducted at the Massachusetts Institute of Technology where she identifies "moves" and "arguments" as two distinct types of reasoning embodied by sketching: the "move" being a tangible proposition within the process, the "argument" (p. 125) being a conceptual micro-activity within that process. She proposes the notion of interactive imagery, involving a combination of imaging, sketching and resketching, stating "sketching is not merely an act of representation of a preformulated image... it is more often than not a search for such an image" (p. 131). She refers to "seeing as" and "seeing that" (Goldschmidt, 1989, pp. 135-136), when interfacing with sketch material: "seeing as" utilises a Gestalt approach to perceiving the content of sketch images to develop ideas. "Seeing that" by contrast, refers to the recording of mental imagery in order to analyse it. She also refers to creative leaps within the mind of the designer, "one in which the distance in time or place among relevant moves is too great to track" (Goldschmidt, 1992, p. 200).

The development of thought supported by sketching is also considered by Goel (1995) who identifies lateral transformations during the creative shift to alternative concepts: “... when a new idea is generated, a number of variations of it quickly follow. The variations expand on the problem space... the 
exploration and transformation of ideas is happening on the paper in front of one's eyes as the subject moves from sketch to sketch" (p. 200).

\section{Establishing the presence of SI}

The immense value that sketches and the sketching process afford successful design is well supported by the literature and this together with initial interview data is presented here to establish sketch inhibition as a phenomenon beyond one of empirical. This is structured according to Booth et al.'s (2016) initial taxonomy into three specific types:

Individual inhibition:

- Intellectual inhibition - the lack of understanding of the benefit that sketching provides the design process,

- Skill-set inhibition - the lack of expertise and experience needed to engage with sketching effectively,

- Personal inhibition - impairment of creative flow due to the need of perfectionism or not being in the right frame of mind.

\section{Social inhibition:}

- Social and comparative inhibition - the fear of being judged unfavourably by others during the process of creating sketch material, and,

- Social loafing - a lack of input in a group situation, either through fear of judgement or laziness.

Technological inhibition: the prevalence of technology resulting in a disinterest in analogue ideation tools. In addition to this, the research also suggests that educational issues are a causal factor in sketch inhibition among undergraduates.

\subsection{Individual inhibition}

The interviews with design undergraduates suggest many have little idea of the creative and cognitive processes they engage with. Inhibition results in a "process (that) is lacking and uninsightful" (Kindleysides, 2017), and offering few developed ideas (Leblanc, 2017). Inhibited students present very little sketch development or, by contrast, small and highly rendered sketches demonstrating little analysis and high levels of self-consciousness in their production. This can lead to "butt ugly" (Powell, M., 2017) designs demonstrating "poor proportional judgment" (ibid) and clear evidence of design fixation. They are also inclined to produce sketchbooks containing prolific if irrelevant development work, or "wallpaper instead of real ideas" (Vessey, 2017).

Leblanc (2015) refers to fear-based inhibition, as do Booth et al. (2016), and posits the belief that experimentation and failure - a normal part of the design process - are somehow unacceptable because: "the development process is widely misunderstood or inaccessible" (Leblanc, 2015, p. 2). She suggests that because of such fear, as soon as students have a suggestion of a concept they rush into the visualisation process, omitting essential stages of evaluation and refinement. Observation of studio sessions prior to this study demonstrate that students with sketch inhibition tend use a very restricted set of materials, will often favour pencil over other tools, and make use of erasers than their more fluent peers. It also suggests that students with a greater understanding of sketch processes combined with competence in their use are more likely to continue using them.

\subsection{Social inhibition}

The rise of social media together with the culture of instant gratification has led to a shift in student attitudes towards learning. "Millennials experience and expect immediacy" (Skiba, 2005, p. 370). This appears to affect their mind-sets when confronted with learning new skills that push them out of their comfort zone: "a lot of people sort of say, 'I'd love to be able to sketch,' and I say, 'well, you can if you try hard enough,' but there seems to be an unwillingness to spend the time" (Mawford, 2016). Sketch inhibition can result in absenteeism from studio sessions or refusal to engage with activities. Fitton (2016) observes: "they just pretend they're doing it, don't do it, or do just a part of it...sometimes they stop coming to their sessions." Additionally, "they will go to the extent of not having produced any sketching 
and not coming into tutorials because they're embarrassed...then they present their sketchbooks and we can have students who have as few as four pages of drawings" (Fitton, 2016). This was observed during a recent studio session: the most inhibited students finding reasons to leave the studio or not engage with the activity. Studio situations also offer opportunities for sketch inhibited students to hide behind those they perceive to be stronger at sketching. Farzaneh et al. (2012, from Diehl and Stroebe, 1987) refer to group activities being subject to social loafing or free-riding by weaker students.

Vessey (2017) and Powell, D. (2017) also observe that children are no longer drawing as they were a generation ago, preferring to spend time with technology-based activities instead, creating a "fear of the white paper" (Bramston, 2017). This in turn is resulting in a demonstrable "lack of maturity" (Phillips, 2017) in design thinking and output.

\subsection{Technological inhibition}

Jonathan Ive, head designer at Apple stated, "students (are) being taught to use computer programs to make renderings that could "make a dreadful design look really palatable" (from Winston (2014). "People don't necessarily use the word "draw," or what we would think of a drawing activity any more in their lives...over the last 15 years...reliance (is) on software to provide the answers rather than deep thinking process" (Mawford, 2016). Additionally, Seligman, (quoted by Furness, 2016) states that "students are now 'more likely to have a blog than a sketchbook." "

The observations support this view - that software cannot replace the complex cognitive activities of effective concept development. During a recent studio session with undergraduates, many struggled to produce any sketch-based ideation. As soon as they were permitted to do so, they dashed towards the available Macs to develop their concepts. These ranged from naïve repeated elements, all straight-linebased, to those containing CAD blocks found online. Some looked impressive, but all evidenced an inability to develop complex ideas combined with a CAD aesthetic. The problem of inexperience is visited by Coyne et al. (2002): "if you only know how to draw a box, your building will be a box, and if you know how to design anything on the computer you can design anything" (p. 270). Kindleysides confirms that the design ability of graduates is governed by their "knowledge of software" (2017).

Leblanc (2015) acknowledges their considerable value, but also laments the prevalence of digital methods in design, "students are judged by their skills with these tools rather than their creativity or problem-solving ability. This unfortunately nurtures the misconception of design as an aesthetic gesture rather than an intellectual, creative thought process that helps solve problems and drive innovation" (p. 5). The essential processes of design embodied by its practical activities have been replaced with what she perceives as "more gratifying digital tools" (p. 2). Tutors have also been victim of what appear to be beautifully rendered images of students' final designs, only to discover that they contain little more than the primitive shapes: 'if tools embellish irrelevant ideas, camouflage problems and give students a false sense of accomplishment - or worse, are mistaken for "good design" - then they may need to be called into question,' (Leblanc, 2015, p. 6).

\subsection{Educational inhibition}

Leblanc (2015) highlights the lack of understanding of the sketching tool at institution level. "We continually observe students struggle with the creative process...exploring and developing ideas into mature designs. Many see sketching only as a means of visualisation and rarely know how to use it as a creative thinking tool" (p. 1).

Downs (2017) believes that institutions and educators fail to understand the difference between the design process and the presentation of designs. Bramston (2017) notes reliance on over-refinement and a fine art approach that continues into higher education, observing student sketchbooks filled with notes and highly polished drawings that fail to demonstrate the thinking behind the ideas. Stacey (2016) bemoans the result-oriented, (as opposed to process-oriented), assessment of pupils' work in primary and secondary education. He believes this leads to a fear-based mentality among students within higher education. Mis-conceptions about the purpose of sketching appear to affect student relationships with the process: "...they think it has to be the most amazing Leonardo da Vinci-type sketch, instead of a working idea, a methodology to discuss your ideas from your head, and it doesn't have to be perfect" (Fitton, 2016). 
Powell, M. (2017) suggests the death of the UK government art school's "systematic approach" and rigidity of pedagogy in drawing education has left students to find their own way, without success. He suggests that drawing tutors lack the skills their peers would have had during the reign of the art school and as a result, are unable to pass on effective tuition to their students. He also believes that tutors who can't draw do not value the process and will fail to instil the practice and enthusiasm for it in their students.

The challenging relationship between the disciplines of fine art and design plays a role in sketch inhibition, Schenk (2005) commenting that the teaching of drawing is inconsistent with the needs of designers; it is often based on fine art practice as opposed to developing a visual syntax to enhance the mental processes required by the designer.

Lambert and Firth (2006) suggest the expansion in design course offerings from higher education is partly responsible for a decline in sketch fluency. They observe a reduction in competition for places which has led to students being recruited where they would previously not have been considered. Vessey (2017) also notes that much larger cohort sizes and a reduction in one-to-one time tuition time has left students without the necessary mentoring they need to acquire a good level of sketching ability.

\section{Development of a conceptual framework for management of sketch inhibition}

During analysis of the literature and initial interview data, emergent themes were identified that provided foundation for the initial structure for a management proposal. Parent/meta-themes were developed into further child/micro-themes using Nvivo as a tool for analysis. Conceptual tools for this purpose also included mind-mapping techniques to identify linear and axial relationships between themes and from there an initial structure began to emerge. This is modelled in Figure 3.

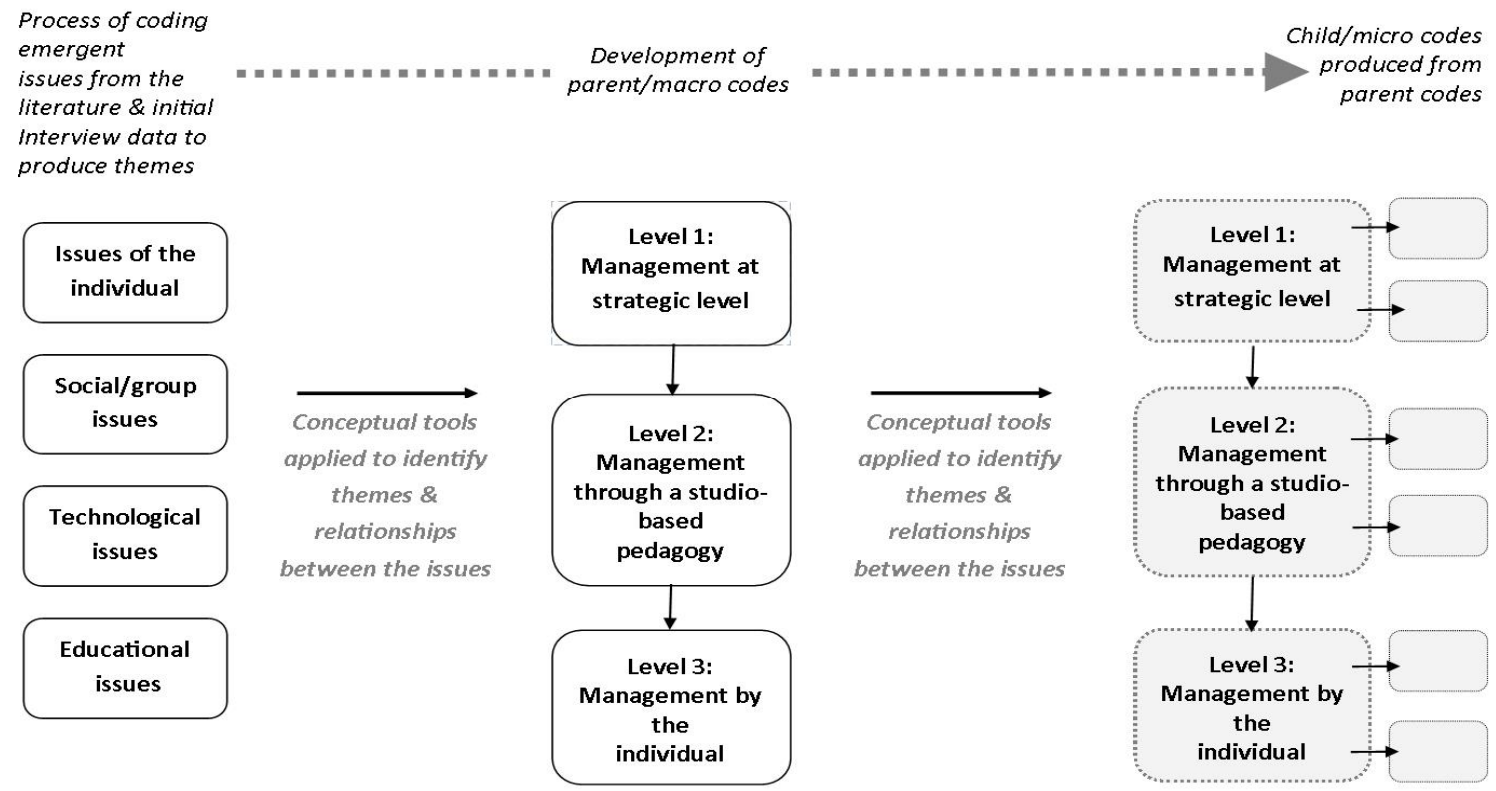

Figure 3. Model to show identification and development of emergent themes from the data using conceptual tools

The conceptual framework that follows presents a formative, indicative management tool and is intended for discussion. A summative framework will be afforded only once the research has reached saturation in due course.

\section{The current propositional management tool}

Though it is anticipated that further issues will emerge during the research, an initial framework has been developed. This is structured into a three-part framework based on identification of the major themes and is presented as a top-down model, although this is subject to testing: 1) Management at 
strategic level within higher education institutions, 2) Management through studio-based pedagogy, and, 3) Management through the attitude of the individual:

Level 1: Management at the strategic level within higher education institutions: The need for greater consultation between higher education institutions and industry during the development stage of courses: Phillips (2017) states that institutions often put their revenue before the relevance of courses to industry. He believes this dialogue could avoid creating "pseudo product design courses."

Raising the profile of sketching as a fundamental design development tool: Cross (2001) cites Ullman et al. (1990), Kavakli et al. (1998) McGown et al. (1998) and Verstijnena et al. (1998) in reinforcing sketching as the most important tool for the effective designer. Vessey (2017) states that students are currently not given enough time to explore their ideas via sketching, their timetables being taken up with learning technical skills useful to the later stages of the design process.

Greater separation between teaching of the design and visualisation stages of design, in order to avoid the blurring of disciplines (according to Powell, M., 2017). This would reduce incidences of students confusing design with visualisation, producing what Vessey (2017) describes as rudimentary shapes that are easy to model and given glossy finishes using CAD tools, but that have little design value.

A revision of recruitment procedures to include observation of applicants sketching ability. According to Powell, M. (2017) many institutions rely on portfolio and interview when recruiting, but an additional sketching task would enable tutors to observe the attitude, understanding and cognitive processes of students while engaged in that activity. It would also serve to reduce instances of fraud (Powell, D., 2017) where students attempt to pass of the work of others in order to gain places.

\section{Level 2: Management through studio-based pedagogy:}

Engagement in mark-making activities: this has been observed to reduce anxiety of inhibited students. This is endorsed by Booth et al. (2016) "warming-up helps engineers (be) less stressed when generating ideas" (p. 8). Booth (2017), and Leblanc (2017) both believe that production of quantity reduces anxiety. Leblanc suggests at least one hundred sketches which forces creative emergence necessary for the development of new ideas, whereas Booth suggests that around fifteen is ample before the reduction in creativity is observed. Booth et al. (2017) suggest that producing sketches at speed reduces the anxiety surrounding their aesthetic. They suggest $28-30$ mins at a time is ample before the brain begins to lose efficiency.

The use of appropriate materials: Bramston (2017) states that architectural pens are a "disaster" as they encourage preciousness. Schenk (2017) recommends big pots of paint and "cheapness" of paper to encourage fluency; Bramston (2017) further endorses the benefits of large paper sizes. The sketchbook is not favoured by Vessey or Schenk: its high quality paper and bound format creates anxiety over spoiling the entire book if a bad sketch is produced Schenk (2017).

Group activities involving sketch sharing: these "reduce ownership" (Bramston, 2017) that leads to inhibition. During observation of studio sessions, students initially presented resistance at having to pass on their sketches to peers, but afterwards expressed enjoyment at the process.

The use of 3D materials and processes to support ideation. Both Fitton (2016) and Bramston (2017) suggest that this can enable development of concepts among students who struggle to ideate using $2 \mathrm{D}$ tools. Fitton (2016) suggests using model-making as a method for students to see their ideas and to then make observations sketches of those ideas to facilitate their development.

Engaging in "purposeful observation" within their own discipline (Schenk, 2017) to improve awareness of the user and environment, (as opposed to fine-art based observation). This enables students to improve their ability and expand their frame of reference within their disciplines without creating pressure to produce an aesthetically pleasing artefact.

In addition to this, a theoretical grounding in the processes that sketching supports including Gestalt and perceptual organisation (Leblanc, 2015), the micro-activities of sketching, cognitive processes and language would be potentially beneficial.

Level 3: Management through the individual: Importance of individual motivation and will to succeed is not to be underestimated: Leblanc (2015) believes that student attitudes are important to the 
management of their own sketch inhibition: "Those with high ambitions and strong self-motivation manage to overcome the deficit; others learn to mask their lack of skills in one area by developing others," (p. 2). Downs (2017) advocates a questionnaire during the recruitment process to establish levels of motivation and maturity of the applicant. This kind of information would offer assessment of students' outside interests and act as an indicator of their potential.

\section{Further work}

Currently, the initial Level 2 pedagogical framework is being developed and will undergo testing in a studio environment. Level 1 is as yet untested and Level 3 can only be addressed by implementing the framework at Level 1 - both these levels will require feedback from peers at institution and industry level to establish their potential benefits. Although beyond the scope of this current study, an interpretive phenomenological study of those suffering from sketch inhibition may identify the deeper issues of sketch inhibition among sufferers and support the development and testing of the pedagogic framework.

\section{References}

Akin, Ö. and Akin, C. (1996), "Frames of reference in architectural design: analysing the hyper-acclamation (Aha!)”, Design Studies, Vol. 17 No. 4, pp. 341-361. https://doi.org/10.1016/S0142-694X(96)00024-5

Alexander, C. (1964), Notes on the Synthesis of Form, MIT Press.

Atilola, O., Tomko, M. and Linsey, J.S. (2016), "The effects of representation on idea generation and design fixation: A study comparing sketches and function trees", Design Studies, Vol. 42, pp. 110-136. https://doi.org/10.1016/j.destud.2015.10.005

Bagnall, J. and Koberg, D. (1990), Universal Traveller, 2nd ed., Crisp Publications.

Barthes, R. (1977), Image, Music, Text, Clays, St Ives, UK.

Bilda, Z. and Gero, J. (2005), "Does sketching off-load visuo-spatial working memory?", Studying Designers, Vol. 5, pp. 145-160.

Bilda, Z., Gero, J. and Purcell, T. (2006), "To sketch or not to sketch? That is the question”, Design Studies, Vol. 27 No. 5, pp. 587-613. https://doi.org/10.1016/j.destud.2006.02.002

Booth, J.W. (2017), Interview, Yale University, USA.

Booth, J.W., Taborda, E.A., Ramani, K. and Reid, T. (2016), "Interventions for teaching sketching skills and reducing inhibition for novice engineering designers", Design Studies, Vol. 43, pp. 1-23. https://doi.org/10.1016/j.destud.2015.11.002

Bramston, D. (2017), Interview, University of Lincoln.

Cash, P. and Kreye, M.E. (2017), "Uncertainty Driven Action (UDA) model: A foundation for unifying perspectives on design activity", Design Science, Vol. 3. https://doi.org/10.1017/dsj.2017.28

Coyne, R., Park, H. and Wiszniewski, D. (2002), "Design devices: digital drawing and the pursuit of difference", Philosophy of Design, Vol. 23 No. 3, pp. 263-286. https://doi.org/10.1016/S0142-694X(01)00038-2

Crismond, D. and Adams, R. (2012), "The Informed Design Teaching and Learning Matrix", Journal of Engineering Design, Vol. 101 No. 4, pp. 738-797. https://doi.org/10.1002/j.2168-9830.2012.tb01127.x

Cross, N. (1999), "Natural intelligence in design", Design Studies, Vol. 20 No. 1, pp. $25-39$. https://doi.org/10.1016/S0142-694X(98)00026-X

Cross, N. (2000), Engineering Design Methods: Strategies for Product Design, 3rd ed., John Wiley and Sons.

Cross, N. (2001), "Design cognition: Results from protocol and other empirical studies of design activity", In: Eastman, C., Newstatter, W. and McCracken, M. (Eds.), Design Knowing and Learning: Cognition in Design Education, Elsevier, Oxford, pp. 79-103.

Diehl, M. and Stroebe, W. (1987), "Productivity loss in brainstorming groups: Toward the solution of a riddle", Journal of Personality and Social Psychology, Vol. 53 No. 3, pp. 497-509. https://doi.org/10.1037/00223514.53.3.497

Downs, S. (2017), Interview, Loughborough University.

Dubberly, H. (2004), How do you design? A compendium of models, Dubberly Design Office.

Eckert, C.M., Blackwell, A.F., Bucciarelli, L.L. and Earl, C.F. (2010), "Shared conversations across design", Design Issues, Vol. 26 No. 3, pp. 27-39. https:// doi.org/10.1162/DESI_a_00027

Farzaneh, H.H., Kaiser, M.K. and Lindemann, U. (2012), "Creative processes in groups-relating communication, cognitive processes, and solution ideas", Proceedings of the 2nd International Conference on Design Creativity, Glasgow, UK, September 18-20, 2012, pp. 13-22.

Faulkner, J. (2017), Interview, Haley Sharpe Design.

Ferguson, E. (1992), Engineering and the Mind's Eye, MIT Press, Cambridge, MA. 
Fish, J. and Scrivener, S. (1990), “Amplifying the mind's eye: sketching and visual cognition”, Leonardo, Vol. 23 No. 1, pp. 117-126. https://doi.org/10.2307/1578475

Fitton, R. (2016), Interview, De Montfort University, Leicester.

Furness, H. (2016), British Museum launches drawing exhibition as art students 'more likely to have a blog than a sketchbook'. [online] Daily Telegraph. Available at: https:/www.telegraph.co.uk/news/2016/08/07/britishmuseum-launches-drawing-exhibition-as-art-students-more

Glaser, B.G. (1992), Emergence vs forcing: Basics of grounded theory analysis, Sociology Press.

Goel, V. (1995), Sketches of Thought: A Study of the Role of Sketching in Design Problem-Solving and its Implications for the Computational Theory of Mind, $\mathrm{PhD}$ thesis, University of California, Berkeley.

Goldschmidt, G. (1989), "Sketching in Design: Past, Present and Future", Proceedings of Environmental Design Research Association, North Carolina State University School of Design, USA, pp. 132-138.

Goldschmidt, G. (1991), "The Dialectics of Sketching”, Creativity Research Journal, Vol. 1 No. 2, pp. $123-143$. https://doi.org/10.1080/10400419109534381

Goldschmidt, G. (1992), "Serial Sketching: Visual Problem-Solving in Designing”, Cybernetics and Systems, Vol. 23 No. 2, pp. 191-219. https://doi.org/10.1080/01969729208927457

Goldschmidt, G. (2003), “The Backtalk of Self-Generated Sketches”, Design Issues, Vol. 19 No. 1, pp. $72-88$. https://doi.org/10.1162/074793603762667728

Goldschmidt, G. (2014), Linkography: unfolding the design process, MIT Press.

Kavakli, M., Scrivener, S.R. and Ball, L.J. (1998), "Structure in idea sketching behaviour", Design Studies, Vol. 19 No. 4, pp. 485-517. http://doi.org/10.1016/S0142-694X(98)00012-X

Kindleysides, J. (2017), Interview, Checkland Kindleysides.

Lambert, I. and Firth, R. (2006), "Pencils Don't Crash", Proceedings of EandDPE 2006, the 8th International Conference on Engineering and Product Design Education, Salzburg, Austria, September 7-8, 2006, pp. 1-6.

Laseau, P. (2001), Graphic thinking for architects and designers, John Wiley and Sons, UK.

Leblanc, T. (2015), "Sketching as a Thinking Process", Proceedings of the 17th International Conference on Engineering and Product Design Education (EandPDE15), Loughborough, UK, September 3-4, 2015.

Leblanc, T. (2017), Interview, University of Montreal, Canada.

Mawford, J. (2016), Interview, Z-Solutions, Suffolk.

McGown, A., Green, G. and Rodgers, P.A. (1998), "Visible ideas: information patterns of conceptual sketch activity”, Design Studies, Vol. 19 No. 4, pp. 431-453. https://doi.org/10.1016/S0142-694X(98)00013-1

Pei, E., Campbell, I. and Evans, M. (2011), "A taxonomic classification of visual design representations used by industrial designers and engineering designers", The Design Journal, Vol. 14, pp. 64-91. https://doi.org/10.2752/175630610X12877385838803

Phillips, P. (2017), Interview, Transparent Product Design.

Powell, D. (2017), Interview, Partner, Seymour Powell.

Powell, M. (2017), Interview, De Montfort University, Leicester.

Schenk, P. (2005), "The Why and How of Drawing: A 20 Year Shift in Design Procedures and Priorities", Proceedings of the European Academy of Design Conference, Bremen, Germany, European Academy of Design, pp. 1-11.

Schenk, P. (2017), Interview, Heriot-Watt University, Edinburgh.

Schön, D. (1983), The Reflective Practitioner: How Professionals Think in Action, Basic Books, New York.

Schön, D. (1992), "Designing as reflective conversation with the materials of a design situation", Knowledgebased systems, Vol. 5 No. 1, pp. 3-14. https://doi.org/10.1016/0950-7051(92)90020-G

Schön, D. and Wiggins, G.D. (1992), "Kinds of Seeing and their Functions in Designing”, Design Studies, Vol. 13 No. 2, pp. 135-156. https://doi.org/10.1016/0142-694X(92)90268-F

Skiba, D.J. (2005), “The Millennials: have they arrived at your school of nursing?", Nursing Education Perspectives, Vol. 26 No. 1, pp. 370-371.

Stacey, M. (2016), Interview, School of Computer Science and Informatics.

Stones, C. and Cassidy, T. (2010), "Seeing and discovering: how do student designers reinterpret sketches and digital marks during graphic design ideation?”, Design Studies, Vol. 31 No. 5, pp. 439-460. https://doi.org/10.1016/j.destud.2010.05.003

Strauss, A. and Corbin, J.M. (1990), Basics of qualitative research: Grounded theory procedures and techniques, Sage Publications.

Suwa, M. and Tversky, B. (1997), "What do architects and students perceive in their design sketches? A protocol analysis", Design Studies, Vol. 18 No. 4, pp. 385-403. https://doi.org/10.1016/S0142-694X(97)00008-2

Ullman, D.G., Wood, S. and Craig, D. (1990), "The importance of drawing in the mechanical design process", Computers and Graphics, Vol. 14 No. 2, pp. 519-546. https://doi.org/10.1016/0097-8493(90)90037-X 
Verstijnena, I.M., van Leeuwenb, C., Goldschmidt, G., Hamelb, R. and Hennesseya, J.M. (1998), "Sketching and Creative Discovery", Design Studies, Vol. 19 No. 4, pp. 519-546. https://doi.org/10.1016/S0142694X(98)00017-9

Vessey, N. (2017), Interview, WMP Creative.

Wacker, J. (1998), "A definition of theory: research guidelines for different theory-building research methods in operations management", Journal of Operations Management, Vol. 16 No. 4, pp. 361-385. https://doi.org/10.1016/S0272-6963(98)00019-9

Winston, A. (2014), Design Education is Tragic. [online] Dezeen. Available at: https://www.dezeen.com/2014/11/13/design-education-tragic-says-jonathan-ive-apple (accessed 15.11.2017)

Witts, M. (2017), Interview, Sosna Witts Design.

Lisa Thurlow, Part time lecturer and $\mathrm{PhD}$ student

De Montfort University, School of Design

Bethesda, IP31 1RR Bury St. Edmunds, United Kingdom

Email: lisa.thurlow@dmu.ac.uk 